\title{
Infrared SEDs of quasars and radio galaxies: Unification and dust evolution seen by ISO, SCUBA and MAMBO
}

\author{
Martin Haas ${ }^{1}$ \\ ${ }^{1}$ Astronomisches Institut, Ruhr-Universität, 44780 Bochum, Germany
}

\begin{abstract}
Sensitive Infrared Spectral Energy Distributions (SEDs) drawn from the ISO data archive and supplemented with SCUBA and MAMBO observations, provide evidence for the geometric unification of powerful 3CR radio galaxies as "edge-on" quasars. Furthermore, detailed SEDs of 64 Palomar-Green quasars show a diversity of shapes, consistent with the physical evolution of the heating sources and the dust distribution around them.
\end{abstract}

\section{Powerful 3CR radio galaxies and quasars}

\subsection{Introduction and data}

The aim of this project is to test the aspect-angle unification proposed by Barthel (1989): Powerful Fanaroff-Riley FR 2 radio galaxies are "edge-on" quasars viewed at high inclination, so that their nuclei are hidden behind a dust torus. If so, then this torus intercepts the optical-ultraviolet AGN radiation and reemits it in the infrared. Therefore a robust check of the unification is to look for the mid- and far-infrared reemission of the absorbed light from the AGN. A great advantage is that at wavelengths $\lambda>25 \mu \mathrm{m}$ the IR emission is largely optically thin, hence isotropic and independent of the aspect angle. The $3 \mathrm{CR}$ catalogue of radio galaxies and quasars is selected at $178 \mathrm{MHz}$ which measures the isotropic, not-beamed, emission of the radio lobes, hence the $3 \mathrm{CR}$ sources provide a well suited database to test the unification.

While IRAS data of 3CR sources did not allow the drawing of definite conclusions about the unification (Heckman et al. 1992, 1994, Hes, Barthel \& Hoekstra 1995, Hoekstra, Barthel \& Hes 1997), first results on small samples have been derived from ISO data (Haas et al. 1998, van Bemmel et al. 2000, Meisenheimer et al. 2001, Andreani et al. 2002). Here we consider the full ISOPHOT data base of 75 sources in the ISO Data Archive, supplemented by SCUBA archive data and new MAMBO observations. The results are presented in detail by Haas et al. (2004). They refer to 35 good detections, 16 radio galaxies and 19 quasars.

We consider the following two basic source classes: i) the steep spectrum quasars and the BLRGs, henceforth denoted as quasars, and ii) the FR 2 NLRGs, henceforth denoted as galaxies. The strategy to check the unification includes two steps: (1) to show that both the quasars and the galaxies exhibit a high mid- to far-IR luminosity ratio typical for AGNs, and (2) that the isotropic FIR-to-radio luminosity ratio is the same for quasars and galaxies at matched isotropic $178 \mathrm{MHz}$ radio power.

\subsection{Results}

Figure 1 shows SED examples for a quasar, a galaxy and a starburst ULIRG. With regard to the FIR emission, the MIR emission of both the quasar and the galaxy is high, while that of the SB-ULIRG is low. Starbursts typically do not provide such a high $\mathrm{L}_{\mathrm{MIR}} / \mathrm{L}_{\mathrm{FIR}}$ ratio as powerful AGNs do. 

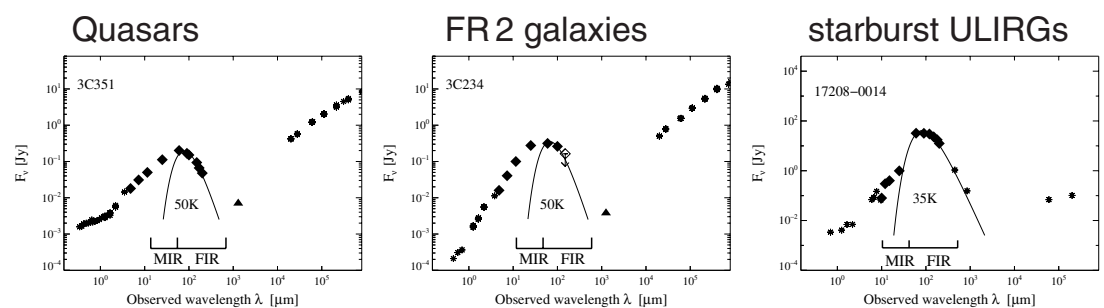

Figure 1. SED examples.
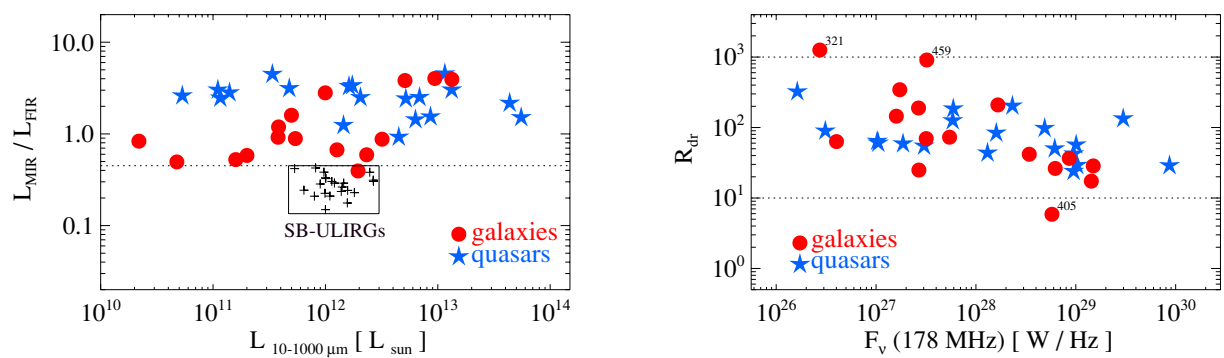

Figure 2. Left: Mid- to far-infrared luminosity ratio versus IR luminosity. Right: Ratio of dust- to radio lobe power $R_{\mathrm{dr}}$ versus radio lobe power.

Figure 2 (left) shows the $\mathrm{L}_{\mathrm{MIR}} / \mathrm{L}_{\mathrm{FIR}}$ ratio for the samples. This ratio is higher for the 3CR sample than for starburst-ULIRGs (from Klaas et al. 2001). This provides evidence for the presence of a powerful AGN in the galaxies as well.

So far we have found evidence for a powerful AGN in both the quasars and the galaxies. In a strict sense, however, the concept of unification requires that for an object drawn from the parent population any isotropic emission remains the same while rotating the viewing angle to its axis. If the unification is valid, then for an ideal sample of parent objects the emitted isotropic FIR dust power should be the same for objects of identical isotropic lobe power. Therefore, we consider $\mathrm{R}_{\mathrm{dr}}$, the ratio of $\nu \mathrm{F}_{\nu}$ at FIR wavelength 70 $\mu \mathrm{m}$ and $\nu \mathrm{F}_{\nu}$ at radio frequency $178 \mathrm{MHz}$.

Figure 2 (right) shows $\mathrm{R}_{\mathrm{dr}}$ versus the $178 \mathrm{MHz}$ radio lobe power. All along the range of the $178 \mathrm{MHz}$ radio lobe power, the distribution of $\mathrm{R}_{\mathrm{dr}}$ for the quasars is strikingly similar as that of the galaxies. This provides clear evidence in favour of geometric unification. Nevertheless, there is a considerable dispersion in the dust/lobe power ratio, which points toward the additional influence of the environment (3C 405) and evolution (3C 321 and 3C 459) of the sources.

\section{Evolution of the dust emission of PG quasars}

\subsection{Introduction and data}

Extending the Sander et al. (1988) idea, that a quasar is preceded by a dusty ULIRG phase, the dust should not disappear at once and its relicts should trace evolutionary steps among the quasars. While former IR data did not allow the recognition of definite detailed signatures (due to limited sensitivity, wavelength coverage or sample size), here 

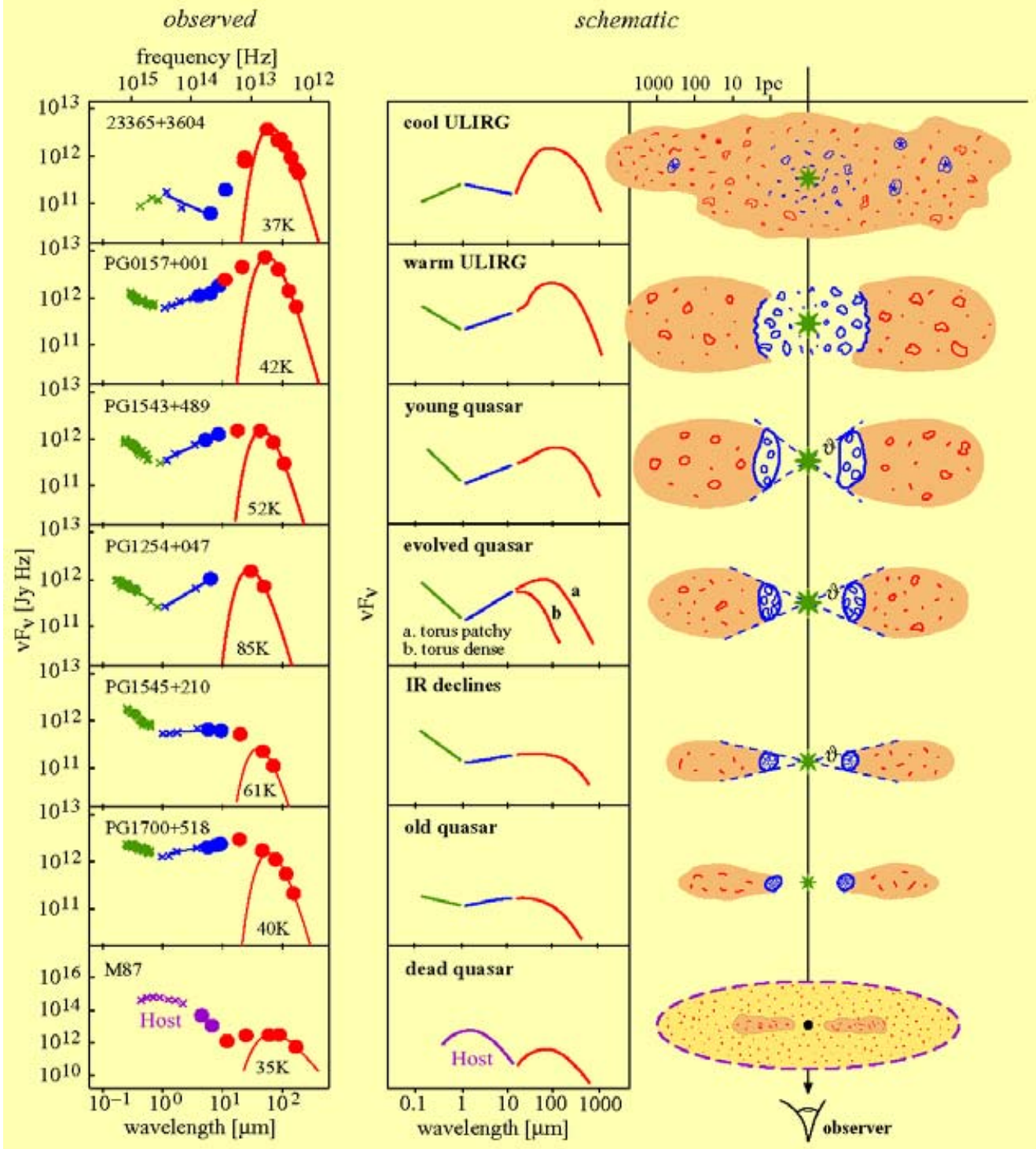

Figure 3. SEDs and scheme of dust distribution surrounding the AGN.

we show that such signatures can be identified for the first time in the sensitive ISO data of 64 PG quasars. The results are presented in detail by Haas et al. (2003).

\subsection{Results}

Actually, the SEDs exhibit a diversity of shapes, as shown in Fig. 3. The observed SEDs reflect both the dust distribution around the heating sources, and the nature of the heating sources as is also illustrated schematically.

Firstly, we consider the physical processes acting on an initially irregular dust distribution. Dissipative cloud collisions and angular momentum constraints lead to the organisation of dust clouds into a torus/disk like configuration. With regard to the emission, the dust which is initially heated by starbursts will be powered more and more 
by the AGN, until the black hole begins to starve. Therefore, it is natural to interpret the diversity of SEDs in terms of evolution. In Fig. 3 the SEDs a arranged along the expectations for such an evolutionary scheme (from top to bottom).

During the evolution, the corresponding SEDs show an initial FIR bump, then an increase in MIR emission and a steepening of the infrared slope, both of which finally also decrease. The AGN strength grows, then stays high and finally declines, as is marked by the size of the $\star$ and shows up in the SEDs by the optical slope.

The PG quasars are practically not extinguished $\left(A_{V}<0.3\right)$. Furthermore, for the full sample of $64 \mathrm{PG}$ quasars, the optical slope $\alpha_{\text {opt }}$ is independent of IR properties like the near- to mid-IR slope $\alpha_{\mathrm{IR}}$. Therefore, with regard to the unified schemes we can assume a nearly face-on view onto the PG quasars.

To conclude, the observed variety of SEDs can be associated with and sorted into physically meaningful classes, which reflect the amount and distribution of the reprocessing dust around the AGN. These classes can naturally be understood as a consequence of evolution of the quasars' dust distribution and heating. Extending the known general evolutionary link between ULIRGs and quasars, the sensitive ISO data allows for establishing the dust evolution even among the PG quasars.

\section{Acknowledgements}

M. H. thanks for support by the Nordrhein-Westfälische Akademie der Wissenschaften, funded by the Federal State and the Federal Republic of Germany.

\section{References}

Andreani, P., Fosbury, R. A. E., van Bemmel, I., \& Freudling, W. 2002, A\&A, 381, 389

Barthel, P. D. 1989, ApJ, 336, 606

Haas, M., et al. 1998, ApJ, 503, L109

Haas, M., et al. 2003, A\&A, 402, 87

Haas, M., et al. 2004, A\&A, submitted

Heckman, T. M., Chambers, K. C., \& Postman, M. 1992, ApJ, 391, 39

Heckman, T. M., O'Dea, C. P., Baum, S. A., \& Laurikainen, E. 1994, ApJ, 428, 65

Hes, R., Barthel, P. D., \& Hoekstra, H. 1995, A\&A, 303, 8

Hoekstra, H., Barthel, P. D., \& Hes, R. 1997, A\&A, 319, 757

Klaas, U., et al. 2001, A\&A, 379, 823

Meisenheimer, K., et al. 2001, A\&A, 372, 719

Sanders, D. B., et al. 1988, ApJ, 325, 74

van Bemmel, I.M., Barthel, P., \& de Graauw, Th. 2000, A\&A, 359, 523 